\title{
Proteomic Profiling of Human Umbilical Cord Blood Serum and its Potential Use in Regenerative Therapies ${ }^{\dagger}$
}

\author{
Ashmitha K. Shetty ${ }^{1, *}$, R. Deveswaran ${ }^{2}$, Ashish Rai ${ }^{3}$, Shivani S. ${ }^{1}$, Manasa B. L. ${ }^{1}$, Saloni B. ${ }^{1}$ \\ 1 Dept. of Pedodontics and Preventive Dentistry, Faculy of Dental Sciences ,MS Ramaiah University of Applied Sciences; \\ ashmitha.pe.ds@msruas.ac.in (A.K.S.); shivanicheluvaraj13@gmail.com (S.S.); manasabgowda1208@gmail.com \\ (M.B.L.); ritu.salonirocks@gmail.com (S.B.); \\ 2 Dept. of Pharmaceutics, Faculty of Pharmacy, MS Ramaiah University of Applied Sciences; \\ deveswaran.ps.ph@msruas.ac.in (R.D.); \\ 3 Dept.of Oral and Maxillofacial Surgery,A.B.Shetty Memorial Institute of Dental Sciences,Nitte University; \\ drashishrai@nitte.edu.in (A.R.); \\ * Correspondence: ashmitha.pe.ds@msruas.ac.in; \\ $\dagger$ Presented at Materials Chemistry and Physics (Materials Chemistry 2020) - International e-Conference
}

Received: 16.09.2020; Revised: 20.09.2020; Accepted: 24.09.2020; Published: 27.09.2020

\begin{abstract}
A few biomedical applications of Human umbilical cord blood serum used for the treatment of blood disorders and ocular therapy have been documented. Umbilical cords are relatively free of bacterial and viral contamination and often discarded after birth. Previous studies on human umbilical cord blood serum have certain factors exhibiting a distinctive differentiation potential and pharmacological influence like to boost anti-inflammatory, analgesic, and wound healing property.

Cord blood can be collected without pain and cryopreserved without causing any changes in the functional properties. Human umbilical cord blood serum has been increasingly used in regenerative medicine because of their ability to treat tissue injury and causing immunomodulatory properties. Human cord blood serum possesses a striking natural resemblance of nutrients required for cell/tissue engineering. Cord blood serum being allogenic in nature, makes it a favorable biomaterial over other autogenous therapies. It contains a number of proteins that are exchanged from the mother to the fetus for growth and development.These exclusive proteins may perhaps participate in the modulation of cell proliferation and differentiation along with accelerated migration of keratinocyte at the wound edge with the generation of the intact epithelium.In the present study, umbilical cord blood serum was obtained from 35 patients and was subjected toMass Spectrometry. A total of 99 proteins were identified in the sample. Functions of proteins were analyzed using the Uniprot database.Thus, understanding the protein functions could give us insight into the regenerative properties of umbilical cord blood serum.
\end{abstract}

Keywords: umbilical cord blood serum; proteome; regeneration; mass spectrometry.

(C) 2020 by the authors. This article is an open-access article distributed under the terms and conditions of the Creative Commons Attribution (CC BY) license (https://creativecommons.org/licenses/by/4.0/).

\section{Funding}

This research was funded by the Division of Reproductive Biology Maternal Health \& Child Health (RBMH\&CH),Indian Council of Medical Research(ICMR)(Project ID:2017-4532).

\section{Acknowledgments}

We acknowledge the Faculty of Pharmacy, M S Ramaiah University of Applied Sciences, for helping in the execution of the study. We thank the Centre of Cellular and Molecular Biology for assisting in Mass Spectrometric analysis and Sterillixir Biotech Ltd, Bangalore, for 
processing the sample. We wish to thank Lakshmi Maternity Hospital, Bangalore, for assisting in sample collection.

\section{Conflicts of Interest}

The authors declare no conflict of interest. 